\title{
Multi-TeV signals of baryogenesis in a Higgs troika model
}

\author{
Hooman Davoudiasl, ${ }^{1, *}$ Ian M. Lewis, ${ }^{2, \dagger}$ and Matthew Sullivan $\oplus^{1, \$}$ \\ ${ }^{1}$ High Energy Theory Group, Physics Department, Brookhaven National Laboratory, \\ Upton, New York 11973, USA \\ ${ }^{2}$ Department of Physics and Astronomy, University of Kansas, Lawrence, Kansas 66045, USA
}

(Received 9 April 2021; accepted 21 June 2021; published 19 July 2021)

\begin{abstract}
A modest extension of the Standard Model by two additional Higgs doublets—-the Higgs troika modelcan provide a well-motivated scenario for successful baryogenesis if neutrinos are Dirac fermions. Adapting the "spontaneous flavor violation" framework, we consider a version of the troika model where light quarks have significant couplings to the new multi-TeV Higgs states. Resonant production of new scalars leading to dijet or top-pair signals are typical predictions of this setup. The initial- and final-state quarks relevant to the collider phenomenology also play a key role in baryogenesis, potentially providing direct access to the relevant early-Universe physics in high-energy experiments. Viable baryogenesis generally prefers some hierarchy of masses between the observed and the postulated Higgs states. We show that there is a complementarity between direct searches at a future $100 \mathrm{TeV} p p$ collider and indirect searches at flavor experiments, with both sensitive to different regions of parameter space relevant for baryogenesis. In particular, measurements of $D-\bar{D}$ mixing at $\mathrm{LHCb}$ probe much of the interesting parameter space. Direct and indirect searches can uncover the new Higgs states up to masses of $\mathcal{O}(10) \mathrm{TeV}$, thereby providing an impressive reach to investigate this model.
\end{abstract}

DOI: 10.1103/PhysRevD.104.015024

\section{INTRODUCTION}

The reason why the visible content of the Universe exists at all still eludes a definitive answer. This is equivalent to asking what gave rise to the observed baryon asymmetry of the Universe (BAU) [1],

$$
\frac{n_{B}}{s} \approx 9 \times 10^{-11},
$$

where $n_{B}$ is the net baryon number density and $s$ is the cosmic entropy density. The answer to this question is generally believed to be furnished by physics beyond the Standard Model (SM). This expectation is implied by the criteria-i.e., the Sakharov conditions [2] — necessary for a successful baryogenesis mechanism that provides the BAU: (i) baryon number $B$ violation, (ii) $C$ and $C P$ violation, and (iii) departure from equilibrium. The SM can meet condition (i) through thermal electroweak processes often referred to as sphalerons at temperatures $T \gtrsim 100 \mathrm{GeV}$

\footnotetext{
*hooman@bnl.gov

ian.lewis@ku.edu

*msullivan1@bnl.gov
}

Published by the American Physical Society under the terms of the Creative Commons Attribution 4.0 International license. Further distribution of this work must maintain attribution to the author(s) and the published article's title, journal citation, and DOI. Funded by SCOAP. before electroweak symmetry is broken. However, conditions (ii) and (iii) are not met to the requisite levels in the $\mathrm{SM}$, and hence extensions of it that lead to viable baryogenesis are well motivated, with numerous ideas having been put forward over the years.

An interesting aspect of baryogenesis through SM sphalerons is its connection with the physics of leptons. That is, these processes can generate the BAU by transforming a primordial asymmetry in $B-L$, where $L$ denotes lepton number. In particular, if a sufficient amount of lepton asymmetry $\Delta L$ is present at $T \gtrsim 100 \mathrm{GeV}$, the sphalerons can provide the observed $\Delta B$. An interesting possibility is offered by leptogenesis [3] through the decay of heavy right-handed Majorana neutrinos, leading to $\Delta L \neq 0$, assuming enough $C P$ violation is present in its interactions. These states are well-motivated components of the seesaw mechanism [4-7] that leads to light Majorana neutrinos. However, generic Majorana leptogenesis employs heavy right-handed states with masses several orders of magnitude above the weak scale, well beyond the reach of direct experimental measurements. This limits their signature mainly to indirect evidence from the observation of lepton number violation in neutrinoless double beta decay, which has so far yielded null results.

In this paper, we consider an extension of the SM that could lead to a viable baryogenesis mechanism, while having potentially observable direct signals at high-energy collider experiments. Our mechanism requires the addition 
of only two extra Higgs doublets, with the same quantum numbers as the SM Higgs doublet, assuming that the SM has already been augmented with right-handed neutrinos that are very likely necessary to endow SM neutrinos with their observed small masses $\lesssim 0.1 \mathrm{eV}$. In fact, as will be discussed below, we find that this Higgs troika model ${ }^{1}[8]$ is quite well motivated if the SM neutrinos are Dirac fermions and seesaw-inspired leptogenesis is not relevant. The troika model can be viewed as a minimal implementation of similar ideas in Refs. [9-11]. For more general discussions of three-Higgs-doublet models, please see Refs. [12-19] and references therein.

In light of the above, here we will assume that neutrinos are Dirac fermions, unlike in our prior work [8], where righthanded neutrinos were taken to be TeV-scale Majorana states. In the present work, we also take a different approach to flavor from that adopted in Ref. [8], where the new Higgs states were assumed responsible for setting up the leptonsector flavor structure. Here, in contrast, all fermion masses are taken to get sourced by one Higgs doublet which corresponds to the SM-like scalar observed at the LHC with a mass of $\approx 125 \mathrm{GeV}$.

In the following, we will allow the new TeV-scale Higgs states to couple with significant strength to light and heavy quarks, thereby providing a possible resonant production channel through quark initial states [20-22], leading to dijet or top-pair final states. This allows for some of the key interactions involved in our baryogenesis mechanism to be tested in high-energy experiments. In particular, we will show that a future hadron collider at a center-of-mass energy $\sqrt{s}=100 \mathrm{TeV}$ will be able to discover the new scalars up to $\mathcal{O}(10) \mathrm{TeV}$ masses. As will be discussed, flavor data provide important constraints on this model and can play a complementary role in probing the parameter space relevant to baryogenesis.

We will next provide a summary of the main features of baryogenesis in the Higgs troika model.

\section{BARYOGENESIS FROM A HIGGS TROIKA}

Here, we will outline some of the key features of the Higgs troika baryogenesis mechanism and refer the interested reader to Ref. [8] for more details. We will also clarify where our present work deviates from the assumptions adopted in that reference, though the main ideas are largely the same. In the troika framework, the observed BAU is generated through the decays of heavy Higgs fields, with masses $\gtrsim 1 \mathrm{TeV}$, from the interference of tree and loop processes, some of which have been illustrated in Fig. 1. In what follows, the three Higgs fields are denoted by $H_{a}$, where $a=1,2,3$, and their interactions with fermions are

\footnotetext{
${ }^{1}$ Troika $=$ A group of three.
}

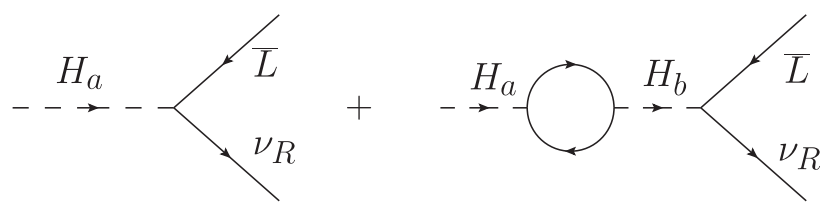

FIG. 1. Tree and one-loop diagrams for lepton number asymmetry from heavy Higgs decays with $a, b=2$, 3. Other one-loop processes that are not enhanced by scalar mass degeneracy are omitted.

$$
\sum_{a=1}^{3} \lambda_{u}^{a} \tilde{H}_{a}^{*} \bar{Q} u+\lambda_{d}^{a} H_{a}^{*} \bar{Q} d+\lambda_{\nu}^{a} \tilde{H}_{a}^{*} \bar{L} \nu_{R}+\lambda_{\ell}^{a} H_{a}^{*} \bar{L} \ell,
$$

where fermion generation indices are suppressed. We will assume that $H_{1}$ is the SM-like doublet giving rise to all fermion and gauge boson masses, and that it contains the $125 \mathrm{GeV}$ boson discovered at the LHC. Here, $H_{a}$ with $a=2,3$ are assumed not to play any role in fermion and gauge boson mass generation, which is a different assumption from that adopted in Ref. [8], as mentioned earlier.

Note that we need at least two Higgs doublets to get a nonzero $C P$-violating phase, necessary for generating an asymmetry $\varepsilon_{a}$ in the decay of the doublet $H_{a}$. However, given our assumption that $H_{1}$ gives mass to Dirac neutrinos, its coupling to the $\bar{L} \nu_{R}$ final state, with $\nu_{R}$ being a right-handed neutrino, is very small: $\sim 10^{-12}$. This obviously makes it impossible to generate a BAU at the level in Eq. (1) if $H_{1}$ is required to contribute. Therefore, we see that in this case, having two additional fields $H_{2,3}$ is a requirement for successful baryogenesis, making a Higgs troika a well-motivated setup. We will take the heavy Higgs fields to be roughly degenerate in mass, at the $\sim 5 \%-10 \%$ level, in which case the one-loop "bubble" diagram shown in Fig. 1 dominates over other one-loop contributions.

The light particles running in the loop could be any of the fermions that couple to $H_{a}$; however, since we will assume that the heavy doublets have $\gtrsim 0.1$ couplings to SM quarks, they will end up giving the dominant one-loop contributions to $\varepsilon_{a}$; other fermion couplings are taken to be smaller by assumption and to satisfy constraints that we will discuss below. In Refs. [21,22], a flavor framework, dubbed "spontaneous flavor violation (SFV)," was proposed to avoid tree-level flavor-changing neutral currents (FCNCs). The SFV framework allows for significant couplings of new Higgs states to light quarks in two-Higgs-doublet models (2HDMs). We will adopt this framework, extended to apply to our troika model, as a general guide for the couplings of $H_{2,3}$ to the quark sector of the SM. We will focus on the "up-type" SFV model [21,22].

Let us consider some of the general constraints that apply to the couplings of $H_{a}$. An important consideration is to make sure that the asymmetry generated through the decay of the Higgs fields is not washed out by scattering 
processes that allow $f \bar{f} \leftrightarrow \bar{\nu}_{R} L$, with $f$ being any fermion in the SM. Let us denote the coupling of $H_{a}$ to $f$ by $\lambda_{f}^{a}$. Following the arguments in Ref. [8], inefficient washout by $H_{1}$ at temperatures $T_{*} \sim 100 \mathrm{GeV}$-characteristic of electroweak symmetry breaking (EWSB)—demands

$$
\lambda_{\nu}^{1} \lambda_{f}^{1} \lesssim 10^{-8}
$$

With our assumption of Dirac neutrino masses from $v \equiv \sqrt{2}\left\langle H_{1}\right\rangle=246 \mathrm{GeV}$, we have $\lambda_{\nu}^{1} \sim 10^{-12}$, and the above relation is trivially satisfied.

The decays of $H_{2,3}$ are assumed to be out of equilibrium - that is, at temperatures below their mass - hence satisfying Sakharov condition (iii) above. The washout condition for the heavy states then has dependence on their masses $m_{a}$ which can suppress thermal processes $\sim m_{a}^{-4}$ [8]. For the rest of this paper, $a=2,3$ unless explicitly specified otherwise. For a reheat temperature near $T_{*}=100 \mathrm{GeV}$, we find

$$
\lambda_{\nu}^{a} \lambda_{f}^{a} \lesssim 2.1 \times 10^{-4}\left(\frac{m_{a}}{10 \mathrm{TeV}}\right)^{2}
$$

assuming that one flavor of quarks and leptons dominates the underlying processes through their couplings to $H_{a}$. In the Appendix, we will outline the derivation of this bound, which is quite consistent with the order-of-magnitude expectation [8].

We now consider requirements on couplings from the generation of a sufficiently large asymmetry $\varepsilon_{a}$. As will be the case later, let us assume that the largest couplings of $H_{a}$ are to quarks $q \in\{Q, u, d\}$, as given in Eq. (2). Quarks will then be the fermions dominating the loop in Fig. 1 and setting the width $\Gamma_{a}$ of the heavy scalars through $H_{a} \rightarrow \bar{q} q$, given by

$$
\Gamma_{a}=\sum_{q} \frac{3\left|\lambda_{q}^{a}\right|^{2}}{16 \pi} m_{a}
$$

To find the regions of parameter space compatible with successful baryogenesis, we follow the formalism developed in Ref. [8] and now include the width of $H_{b}$ [23]. In the on-shell renormalization scheme, the dispersive part of the bubble diagram in Fig. 1 does not contribute to the decay [23]. Hence, we only include the absorptive part of the bubble diagram, and the asymmetry parameter that governs baryogenesis generated by the diagrams in Fig. 1 is

$\varepsilon_{a}=\frac{1}{8 \pi} \frac{\left(m_{b}^{2}-m_{a}^{2}\right) m_{a}^{2}}{\left(m_{b}^{2}-m_{a}^{2}\right)^{2}+m_{b}^{2} \Gamma_{b}^{2}} \frac{\sum_{f=q} N_{c, f} \operatorname{Im}\left(\operatorname{Tr}_{\nu}^{b a} \operatorname{Tr}_{f}^{b a *}\right)}{\sum_{f=q} N_{c, f} \operatorname{Tr}_{f}^{a a}}$,

where $\operatorname{Tr}_{f}^{b a}=\operatorname{Tr}\left[\lambda_{f}^{b \dagger} \lambda_{f}^{a}\right]$.
A rough schematic estimate for $\varepsilon_{a}$ is then given by

$$
\varepsilon_{a} \sim \frac{\left(\lambda_{\nu}^{a}\right)^{2}}{8 \pi} \sin \theta_{f}
$$

where we have assumed that $\mathrm{H}_{2}$ and $\mathrm{H}_{3}$ have the same couplings to fermions. In the above, $\sin \theta_{f} \sim 1$ is assumed and represents the physical $C P$-violating phase contained in the rephasing invariant quantity $\operatorname{Im}\left(\operatorname{Tr}_{\nu}^{b a} \operatorname{Tr}_{f}^{b a *}\right)$ in Eq. (6), necessary to achieve an asymmetry. A short analysis [8], which we recap in the Appendix, can show that a nonthermal production mechanism for $H_{a}$, based on the decay of a heavy modulus of mass $m_{\Phi} \gtrsim 2 m_{a}$, implies that we need

$$
\varepsilon_{a} \gtrsim 3.4 \times 10^{-8}\left(\frac{m_{\Phi}}{20 \mathrm{TeV}}\right)
$$

Then, Eq. (7) requires $\lambda_{\nu}^{a} \gtrsim 10^{-3}$. Hence, the relation (4) can be roughly satisfied for $\lambda_{\nu}^{a} \sim 10^{-3}$ and $\lambda_{q}^{a} \sim 0.1$, with $m_{a} \sim 10 \mathrm{TeV}$, and we could have significant couplings of heavy Higgs states to quarks in the context of the troika baryogenesis.

Note that the constraint from Eq. (7) can be further relaxed for a modest degeneracy $m_{2} \approx m_{3}$ [8], which we will assume in our phenomenological study. Hence, the above conclusions on the size of the quark couplings to $H_{a}$ can be deemed fairly conservative. As will be illustrated later, the above interactions allow for a test of the baryogenesis mechanism, through discovery of the Higgs states, up to masses $\gtrsim 10 \mathrm{TeV}$ at a future $100 \mathrm{TeV} p p$ collider.

\section{FLAVOR MODEL}

As mentioned earlier, we extend the up-type SFV 2HDM developed in Refs. [21,22] for our Yukawa scheme. In the up-type model, the second Higgs doublet couples to uptype quarks proportionally to their SM Yukawa couplings, with a proportionality constant $\xi$. The coupling to downtype quarks, on the other hand, is arbitrary but diagonal in the mass basis, with $\kappa_{d}, \kappa_{s}$, and $\kappa_{b}$ denoting the couplings to down, strange, and bottom quarks, respectively. The second doublet also couples to charged leptons with a strength proportional to their SM Yukawa couplings, with a proportionality constant $\xi^{\ell}$.

We adopt this up-type SFV 2HDM flavor scheme for both the second and third Higgs doublets. However, we also wish to accommodate the Higgs doublets coupling to neutrinos. For this purpose, we additionally add in arbitrary massdiagonal couplings to neutrinos in a similar fashion to how the Higgs doublets couple to down-type quarks. To summarize, the Yukawa matrices for the heavy Higgs doublets $H_{2,3}$ in our model would be given most generally by 


$$
\begin{aligned}
\lambda_{u}^{2,3} & =\xi \lambda_{u}^{1}, \\
\lambda_{d}^{2,3} & =\operatorname{diag}\left(\kappa_{d}, \kappa_{s}, \kappa_{b}\right), \\
\lambda_{\ell}^{2,3} & =\xi^{\ell} \lambda_{\ell}^{1}, \\
\left|\lambda_{\nu}^{2,3}\right| & =\operatorname{diag}\left(\kappa_{\nu_{1}}, \kappa_{\nu_{2}}, \kappa_{\nu_{3}}\right),
\end{aligned}
$$

where $\lambda_{u}^{1}\left(\lambda_{\ell}^{1}\right)$ is the up-type quark (charged lepton) Yukawa matrix for the doublet $H_{1}$. SFV is a specific realization of general flavor alignment [24-29]. So that our modification to the up-type SFV 2HDM will be minimally invasive, most of the couplings in Eq. (9) will be the same or similar for $\mathrm{H}_{2}$ and $\mathrm{H}_{3}$. We will put all the new sources of $C P$ violation into the neutrino Yukawa couplings of $\mathrm{H}_{2}$ and $\mathrm{H}_{3}$. These neutrino couplings $\lambda_{\nu}^{2,3}$ from Eq. (9) will have arbitrary $C P$-violating phases; generating an asymmetry requires a mismatch in the phases between $\lambda_{\nu}^{2}$ and $\lambda_{\nu}^{3}$, as can be seen from Eq. (6).
The $C P$-violating phases also contribute to the electron electric dipole moment (EDM), so we will use our estimate of this contribution from Ref. [8]:

$$
d_{e} \sim \frac{e \lambda_{\nu}^{a 2} m_{e}}{16 \pi^{2} m_{a}^{2}},
$$

assuming an order-1 $C P$-violating phase. This estimate originates from one-loop charged Higgs couplings. We note that a more thorough analysis [30] of the electron EDM shows that $d_{e}$ is proportional to the mass difference between the $C P$ even and odd scalars in the Higgs doublets. In our analysis, we assume that this mass difference is small; hence, Eq. (10) is a conservative estimate of the electron EDM. Even for $\xi^{\ell}=1$, the contribution from the electron Yukawa $\lambda_{e}^{2,3} \sim 10^{-6}$ is strongly suppressed, while the neutrino couplings can be much larger $\lambda_{\nu}^{2,3} \gtrsim 10^{-4}$ and still satisfy the washout condition in Eq. (4). Hence, $\lambda_{\nu}^{a}$ are

FCNC Constraints, $\kappa_{s}=\kappa_{b}=0, \xi=1$

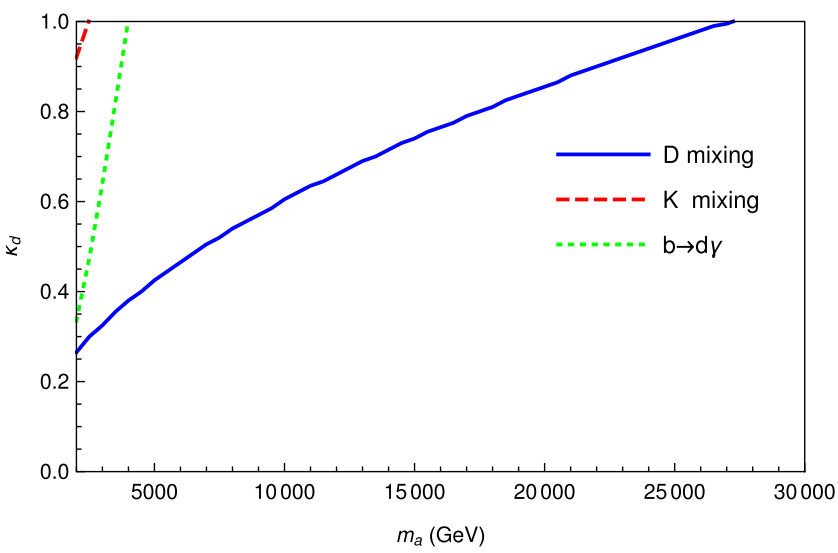

(a)

FCNC Constraints, $\kappa_{s}=\kappa_{b}=0, \xi=0.1$

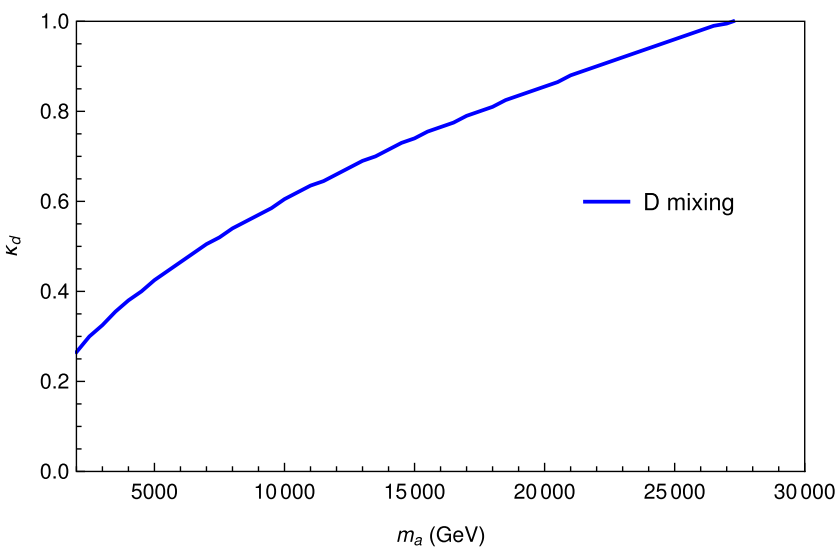

(c)
FCNC Constraints, $K_{s}=K_{b}=K_{d}, \xi=1$

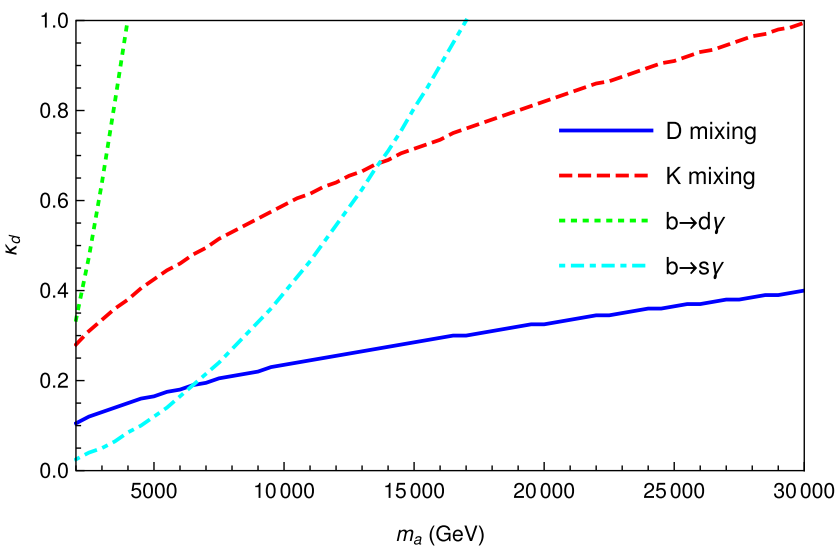

(b)

FCNC Constraints, $\kappa_{s}=\kappa_{b}=\kappa_{d}, \xi=0.1$

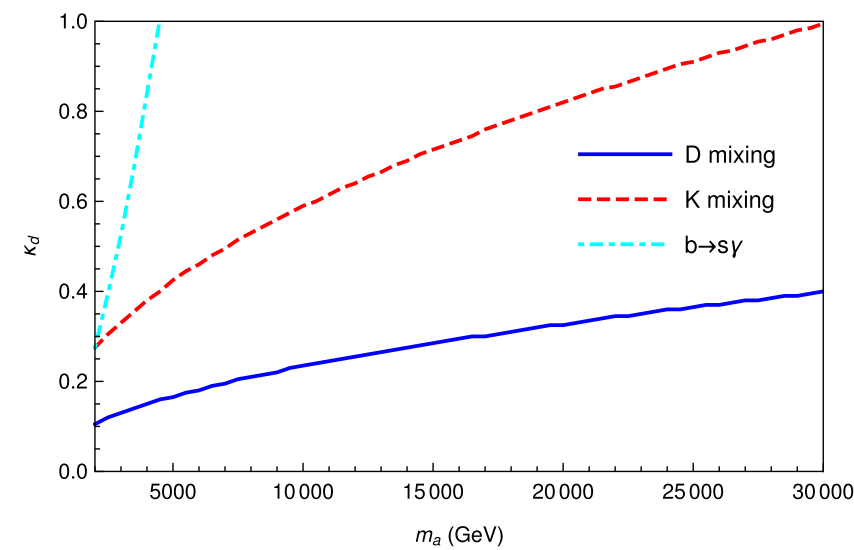

(d)

FIG. 2. Upper bounds from neutral meson mixing and flavor-changing decays on the coupling of $H_{2,3}$ to the down quark, $\kappa_{d}$, for some sample parameter assignments: (a) $\kappa_{s}=\kappa_{b}=0, \xi=1$; (b) $\kappa_{s}=\kappa_{b}=\kappa_{d}, \xi=1$; (c) $\kappa_{s}=\kappa_{b}=0, \xi=0.1$; (d) $\kappa_{s}=\kappa_{b}=\kappa_{d}, \xi=0.1$. The curves represent bounds from $D$-meson mixing (blue solid), $K$-meson mixing (red dashed), $b \rightarrow d \gamma$ decays (green dotted), and $b \rightarrow d \gamma$ decays (cyan dot-dashed). 
the dominant contributions as reflected in Eq. (10). In combination with our washout bounds in Eq. (4), quark couplings of $\kappa_{d} \geq 0.01$ and $\kappa_{s}=\kappa_{b}=\xi=0$, and heavy Higgses with masses in the range of $2-30 \mathrm{TeV}$, we estimate this contribution to the electron EDM will always be at least a factor of 20 below the current electron EDM bound of $d_{e}<1.1 \times 10^{-28} e \mathrm{~cm}$ [31]. Hence, the electron EDM is not constraining.

The SFV scheme suppresses FCNC processes, but experimental bounds are still constraining, especially for lighter Higgses. The relevant experimental bounds come from the flavor-changing decays $b \rightarrow d \gamma$ [32] and $b \rightarrow$ $s \gamma\left(\ell^{+} \ell^{-}\right)$[33], as well as neutral meson mixing for $K-\bar{K}$ [34], $B_{d}-\bar{B}_{d}$ [35], $B_{s}-\bar{B}_{s}$ [35], and $D-\bar{D}$ [36]. We use the formulas presented in Ref. [22] to calculate the contributions to these different processes in our model and have checked that we reproduce their results. Assuming $\mathrm{H}_{2}$ and $\mathrm{H}_{3}$ have identical masses and Yukawa couplings, and taking the alignment limit, we show the relevant limits that these experimental bounds place on the couplings in Fig. 2.

For the multi-TeV masses and Yukawa couplings that we consider, the most constraining measurements are $D-\bar{D}$ mixing and sometimes $b \rightarrow s \gamma\left(\ell^{+} \ell^{-}\right)$for lighter masses. The $D$-meson mixing constraints are largely independent of $\xi \lesssim 1$ due to the dominant contribution of $\kappa_{d}, \kappa_{s}, \kappa_{b}$ via charged Higgs loops. The flavor-changing decay $b \rightarrow d \gamma$ is only the strongest constraint below the mass region we are interested in, but it is still close to being competitive for masses of around $2 \mathrm{TeV}$. The constraints for $B_{s}-\bar{B}_{s}$ and $B_{d}-\bar{B}_{d}$ mixing are constraining only for masses lower than what we consider, but $K-\bar{K}$ mixing constraints are within about a factor of 2 of the stronger $D-\bar{D}$ mixing and $b \rightarrow s \gamma\left(\ell^{+} \ell^{-}\right)$bounds when multiple down-type Yukawa couplings are nonzero. Improved measurements for some of these processes-in particular, $D-\bar{D}$ mixing at $\mathrm{LHCb}$, and also perhaps $K-\bar{K}$ mixing and the $b \rightarrow s \gamma$ and $b \rightarrow d \gamma$ flavor-changing decays - may provide discovery channels for our model in the future. There is also much work going into calculating the SM predictions to greater precision; see Ref. [37] for a recent discussion of the flavor physics prospects of future lattice QCD improvements in combination with current and future experiments.

\section{COLLIDER SIGNATURES}

As mentioned previously, with the couplings in Eq. (9) it is possible to resonantly produce the additional heavy Higgs bosons at hadron colliders via direct couplings to quarks [21,22]. This is unlike traditional Higgs processes, where the Higgs couples so weakly to the light quarks that it must either be produced via loop-level gluon and quark processes, or in multibody final states. Resonant production with light quark initial states greatly enhances the hadron collider reaches.
Assuming the additional heavy Higgses do not mix with the $125 \mathrm{GeV}$ Higgs ("alignment limit"), they will not decay to gauge bosons or the SM-like Higgs pairs. Hence, once a heavy Higgs boson is produced, it will dominantly decay into jets or top quarks. We project bounds from the LHC with $3 \mathrm{ab}^{-1}$ and discovery potential at a $100 \mathrm{TeV} p p$ collider with $3 \mathrm{ab}^{-1}$ and $10 \mathrm{ab}^{-1}$ in the dijet channel. To generate production cross sections, the couplings of Eq. (9) are implemented in MadGraph5_aMC@NLO [38] via FeynRules $[39,40]$. The results of these extrapolations are shown in Fig. 3. The results at the HL-LHC are accomplished via a simple root luminosity scaling of current dijet bounds from ATLAS [41] and CMS [42]. Both ATLAS and CMS present their results as limits on cross section times branching ratio times acceptance. We calculate acceptances using parton level acceptance cuts in MadGraph5_aMC@NLO.

For the $1 \overline{00} \mathrm{TeV}$ collider's $5 \sigma$ discovery reach, we reinterpret the $Z_{B}$ bounds projected in Ref. [43]. Using the same methods above, we implement the $Z_{B}$ model in MadGraph5_aMC@NLO and translate the gauge coupling vs mass limits into dijet cross section limits. The limits and discovery reaches presented in Fig. 3 assume one Higgs doublet with equal charged Higgs mass $m_{H^{+}}$, scalar mass $m_{H}$, and pseudoscalar mass $m_{A}$. With the equal mass and alignment assumptions, the heavy Higgs doublets give vanishing contributions to the $S, T$, and $U$ oblique parameters [44-48]. We would like to make two observations about the equal mass assumption:

(1) The mass differences between the heavy scalar, pseudoscalar, and charged Higgses originate from EWSB. Hence, the mass differences are proportional to the EW vacuum expectation value. For a $10 \mathrm{TeV}$ Higgs at order- 1 couplings in the scalar potential, this is roughly a $0.02 \%-0.03 \%$ mass difference. The decay of a $10 \mathrm{TeV}$ particle will result in jets with an energy of $\sim 5 \mathrm{TeV}$. The jet energy resolution of a jet with energy $5 \mathrm{TeV}$ at a future circular hadron collider is $\sim 3 \%$ [49]. Hence, the reconstructed dijet resonance is expected to have a mass resolution of $\sim 6 \%$, well above the theoretically expected mass difference of $0.02 \%-0.03 \%$. Hence, the resonances would be an overlapping distribution, and our assumption of equal masses is not far from what may realistically be expected at a collider.

(2) The subject of interference between degenerate scalars and pseudoscalars is an interesting one [30]. In the case they are $C P$ eigenstates, they do not interfere, and their rates add incoherently. However, in the model we study, there is $C P$ violation. Hence, the scalar and pseudoscalar can mix via fermion loops. If this mixing is large, interference effects must be accounted for when calculating the production cross section at colliders. These interference effects can be large and negative 


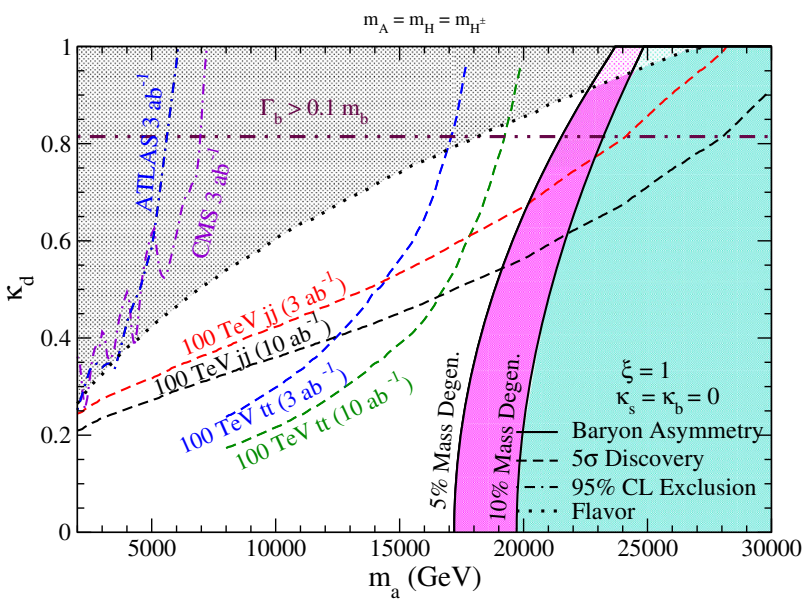

(a)

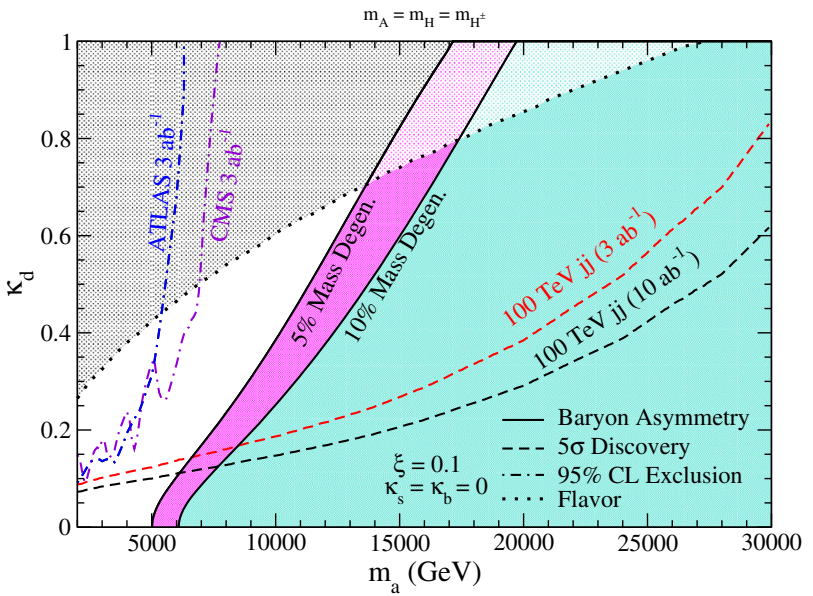

(c)

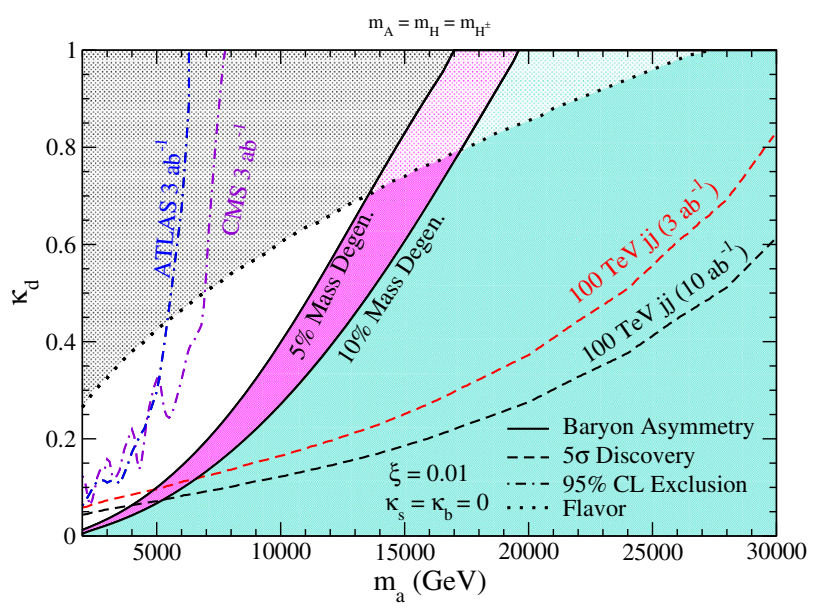

(e)

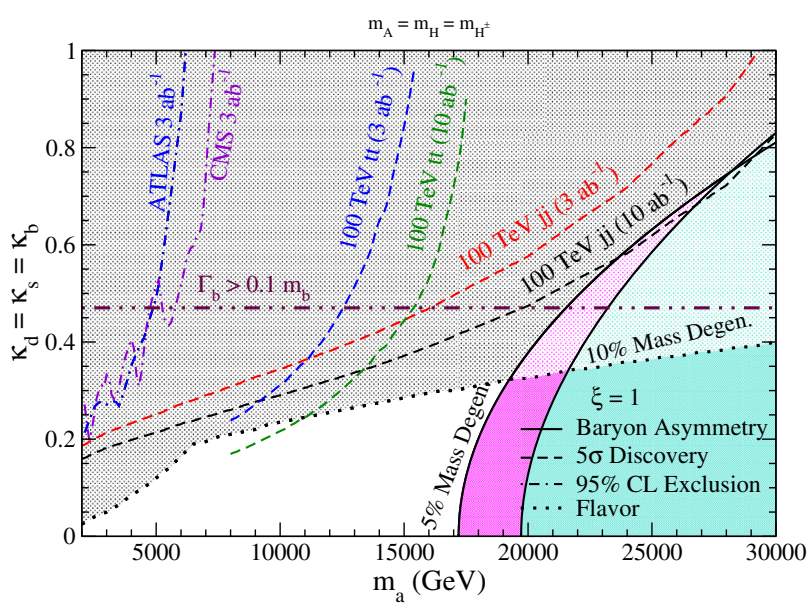

(b)

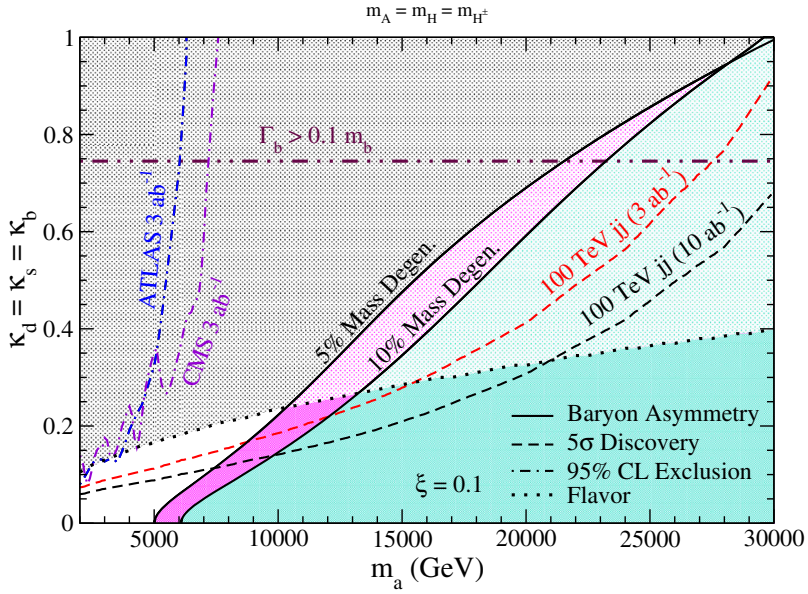

(d)

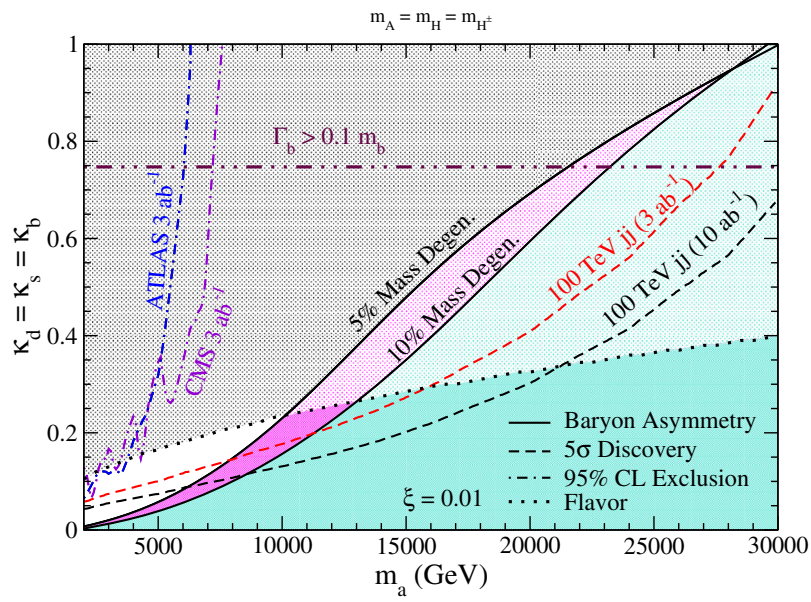

(f)

FIG. 3. Regions above dashed lines are discoverable at $5 \sigma$ in the dijet channel at a $100 \mathrm{TeV}$ collider with $3 \mathrm{ab}^{-1}$ (red) and $10 \mathrm{ab}^{-1}$ (black), and in the $t \bar{t}$ channel with $3 \mathrm{ab}^{-1}$ (blue) and $10 \mathrm{ab}^{-1}$ (green). Regions above dot-dashed lines can be excluded at 95\% at ATLAS (blue) and CMS (violet) with $3 \mathrm{ab}^{-1}$. The regions below the solid lines and color shaded are compatible with successful baryogenesis with a $5 \%$ mass degeneracy $m_{a} / m_{b}=0.95$ (magenta) and a $10 \%$ mass degeneracy $m_{a} / m_{b}=0.9$ (turquoise). Regions above the dotted lines and either gray or shaded in light color are excluded by the flavor bounds presented in Fig. 2. Regions above the maroon dot-dotdashed lines have width-to-mass ratios $\Gamma_{b} / m_{b}$ larger than $10 \%$; we expect our approximation of the decay asymmetry to be good below this line. These are shown in the $\kappa_{d}$ vs Higgs doublet mass, $m_{a}$, plane for (a),(c),(e) $\kappa_{s}=\kappa_{b}=0$; (b),(d),(f) $\kappa_{d}=\kappa_{s}=\kappa_{b}$; (a),(b) $\xi=1$; (c),(d) $\xi=0.1$; and (e),(f) $\xi=0.01$. 
[30], decreasing the rate below the naive factor of 2 . However, in our case we can place the $C P$-violating phase in the neutrino Yukawa couplings. Since the neutrinos couple very weakly to all Higgses, the loop-induced scalar-pseudoscalar coupling is expected to be negligible. Hence, in our calculation of rates we take the optimistic scenario and assume the scalar and pseudoscalar add incoherently. If there is large mixing between the scalar and pseudoscalar, a more careful calculation is warranted.

Finally, in calculating rates at colliders, we use the couplings to physical Higgs bosons in the alignment limit as given in Table 7 of Ref. [22]. ${ }^{2}$

Assuming all quarks contribute to the washout condition, using the flavor structure under consideration given in Eq. (9), and applying the washout condition in Eq. (4), we find

$$
\begin{aligned}
\varepsilon_{a} \lesssim & 1.8 \times 10^{-9}\left(\frac{m_{a}}{10 \mathrm{TeV}}\right)^{4} \frac{\left(m_{b}^{2} / m_{a}^{2}-1\right)}{\left(m_{b}^{2} / m_{a}^{2}-1\right)^{2}+m_{b}^{2} \Gamma_{b}^{2} / m_{a}^{4}} \\
& \times \frac{1}{\kappa_{d}^{2}+\kappa_{s}^{2}+\kappa_{d}^{2}+\xi^{2}} .
\end{aligned}
$$

In the above expression, we have implicitly assumed that there is a physical phase $\theta_{f} \neq 0$, necessary to generate an asymmetry and originating from $\operatorname{Im}\left(\operatorname{Tr}_{\nu}^{b a} \operatorname{Tr}_{f}^{b a *}\right)$ in Eq. (6), with $\left|\sin \theta_{f}\right| \lesssim 1$. We note that as $m_{a} \rightarrow m_{b}$, the width of the intermediate $H_{b}$ in Fig. 1 will regulate the growth of $\varepsilon_{a}$, and further mass degeneracy will not enhance the asymmetry and dominance of the one-loop process shown in the figure.

Since we consider Higgs masses up to $30 \mathrm{TeV}$, the mass of the modulus that decays into the Higgs bosons to create the baryon asymmetry must satisfy $m_{\Phi} \gtrsim 60 \mathrm{TeV}$. From Eq. (8), this translates to a requirement that $\varepsilon_{a} \gtrsim 10^{-7}$; the shaded regions in Fig. 3 are derived using this baryogenesis requirement. For a given Higgs mass and $\xi$, the washout condition in Eq. (4) sets a maximum neutrino coupling, while $\varepsilon_{a} \gtrsim 10^{-7}$ sets a minimum. As $m_{a}$ increases, the coupling $\lambda_{\nu}^{a}$ compatible with washout increases and successful baryogenesis can occur. Hence, for fixed $\xi$, there is a minimum $m_{a}$, typically in the multi-TeV region, required to simultaneously satisfy both washout and asymmetry parameter requirements. For smaller $\xi$, the washout condition is relaxed and smaller Higgs masses are allowed.

Despite needing multi-TeV Higgses, as can be clearly seen in Fig. 3, a $100 \mathrm{TeV} p p$ collider has great potential to discover this scenario. As $\xi$ becomes smaller, the branching ratios into top quark final states are suppressed, enhancing the dijet cross section. Hence, the discovery potential is greater for smaller $\xi$. Reference [43] also has projected

\footnotetext{
${ }^{2}$ We account for a typo in v2 of Ref. [22] and include a missing $1 / \sqrt{2}$ normalization for the neutral scalars in Table 7 .
}

bounds on $t \bar{t}$ resonances at $100 \mathrm{TeV}$. For higher values of $\xi \sim 1$, the discovery reach in the $t \bar{t}$ channel is greater than the dijet channel for Higgs masses below 11-17 TeV. However, as seen in the Figs. 3(a) and 3(b), the dijet searches are still more sensitive to the regions of parameter space that can accommodate baryogenesis. The top pair bounds are not relevant for $\xi=0.01$ and 0.1 , and hence are not shown.

We also superimpose the most stringent flavor bounds from Fig. 2 onto the collider and baryogenesis constraints in Fig. 3. For $\kappa_{s}=\kappa_{b}=0$, a $100 \mathrm{TeV}$ collider always has a greater reach than the searches in flavor physics. However, this is not true for $\kappa_{d}=\kappa_{s}=\kappa_{b}$. In this case, for $\xi=1$ the flavor constraints rule out the parameter regions a $100 \mathrm{TeV}$ collider could discover (with our assumptions, as further discussed below). For $\xi=0.1$ and $\xi=0.01$, there are allowed baryogenesis-compatible regions that a $100 \mathrm{TeV}$ collider could discover for $m_{a} \lesssim 15-20 \mathrm{TeV}$. From this discussion, it is clear that measurements of flavor observables and searches at colliders are sensitive to complementary regions of parameter space. Improvements in $D-\bar{D}$ constraints are especially sensitive to $\kappa_{d}=\kappa_{s}=\kappa_{b}, \xi=1$, and $m_{a} \gtrsim 15-20 \mathrm{TeV}$. A $100 \mathrm{TeV}$ collider has great potential to discover baryogenesis in this model for almost all other parameter regions.

Our calculation of asymmetry parameter depends on the dominance of the bubble diagrams in Fig. 1 over possible triangle diagrams. Hence, we include the maroon dot-dotdashed lines in Fig. 3, where the width of $H_{b}$ is $10 \%$ of its mass. Below these lines, we expect our estimate of $\varepsilon_{a}$ to be valid. If a plot does not include this line, then all regions of parameter space are consistent with $\Gamma_{b}<0.1 m_{b}$. For the $\kappa_{d}=\kappa_{s}=\kappa_{b}$ cases (the right-hand side of Fig. 3), it appears that our calculation is not valid in many regions of parameter space shown. However, we would note that the flavor constraints force our model into regions where $\Gamma_{b}<0.10 m_{b}$, except for a tiny corner of parameter space in Fig. 3(a). Hence, we expect our results to be robust.

Finally, we note that our dijet projections are conservative. It has been assumed that only one Higgs doublet is searched for. However, a distinct prediction of our model is that for successful baryogenesis with $m_{a} \gtrsim \mathcal{O}(1 \mathrm{TeV})$, there is a second Higgs doublet close in mass. For larger mass separations, this could generate a signal of multiple distinct dijet resonances. If the separation between the Higgs bosons masses is less than the detector jet energy resolution but greater than the Higgs widths, our signal may appear as a broad resonance with twice the signal cross section. This would increase our cross section by up to a factor of 2 and coupling reach by up to a factor of $\sim \sqrt{2}$. If the Higgs mass separation is less than the Higgs widths, we would expect a coherent enhancement, since the two Higgs doublets have the same quantum numbers. This would increase our cross section up to a factor of 4 and coupling reach up to a factor of $\sim 2$. The precise details of how to search for these scenarios 
depend intimately on the Higgs mass spectrum, the magnitude of the quark couplings and Higgs widths, and the resolution of future detectors. However, even in the conservative scenario considered here, a $100 \mathrm{TeV} p p$ collider has great potential to discover the baryogenesis mechanism we have proposed.

The factor-of- 2 sensitivity enhancement from constructive coherent interference between the heavy Higgses will increase the sensitivity of ATLAS and CMS to our baryogenesis mechanism. To satisfy Eq. (11), $\varepsilon_{a} \gtrsim 10^{-7}$, and to have the mass difference between the heavy Higgs bosons be less than the width, we find the width-to-mass ratio must be percent level or smaller. Given that level of degeneracy, ATLAS and CMS may be sensitive to our baryogenesis mechanism for $m_{a} \lesssim 5-7 \mathrm{TeV}$ and $\xi \lesssim 0.1$.

\section{CONCLUSIONS}

In this work, we considered the Higgs troika baryogenesis mechanism, assuming that the heavy scalar states from new Higgs doublets couple to light quarks with significant strengths. This setup can avoid large flavor violation effects, assuming the "spontaneous flavor violation" framework, which we adapted for our proposal as a general guide for the new Yukawa couplings. The light quark couplings to the two new Higgs doublets allow for their resonant production. These interactions are also key components of the proposed baryogenesis mechanism, which favors a hierarchy of masses between the SM-like Higgs and the new doublets to avoid the washout of baryon number.

We find that direct searches at colliders and indirect searches from flavor physics are sensitive to different regions of parameter space that are consistent with successful baryogenesis. If only one down-type quark coupling is nonzero, then a $100 \mathrm{TeV}$ collider will be the main discovery channel, and with $3-10 \mathrm{ab}^{-1}$ of integrated luminosity can probe significant regions of the baryogenesis parameter space. When all down-type quark couplings are nonzero and equal, searches in flavor space are sensitive to our baryogenesis mechanism in regions of parameter space complementary to direct searches at a $100 \mathrm{TeV}$. Finally, if the mass difference between the heavy Higgses is less than their widths, the production and decay cross sections are coherently enhanced. In this case, the LHC has the potential to probe baryogenesis in the Higgs troika model for masses $\lesssim 5-7 \mathrm{TeV}$ and width-to-mass ratios at the percent level or smaller. Hence, current and envisioned future collider experiments as well as improvements in bounds on flavor observables can potentially probe the new states and examine their relevance to the proposed processes for generating the baryon asymmetry of the Universe.

\section{ACKNOWLEDGMENTS}

We thank S. Homiller for helpful communication. H. D. and M. S. are supported by the United States Department of
Energy under Contract No. DE-SC0012704. I. M. L. is supported in part by the United States Department of Energy under Contract No. DE-SC0017988. The data to reproduce the plots are available upon request.

\section{APPENDIX}

\section{Washout rate estimation}

Here, we outline our approach to the estimation of the lepton number asymmetry $\Delta L$ washout rate. Since the troika baryogenesis mechanism relies on the amount of $\Delta L$ generated via heavy Higgs doublet decays, one needs to make sure that its washout is not efficient once the reheat temperature $T_{\text {rh }}$ has been established. For a viable scenario, $T_{\text {rh }}$ needs to be at or above the electroweak phase transition temperature $T_{*} \sim 100 \mathrm{GeV}$, in order to have active sphaleron processes necessary to generate the baryon number from $\Delta L$.

In order to determine the efficiency of the washout rate $\Gamma_{\text {wo }}(T)$ at temperature $T$, we will consider the ratio (see, for example, Ref. [50])

$$
\frac{\Gamma_{\mathrm{wo}}(T)}{\mathcal{H}(T)}=\frac{\left\langle\sigma_{\mathrm{wo}}\right\rangle}{2 n_{\gamma}(T) \mathcal{H}(T)} \prod_{i} n_{i}(T),
$$

where $\mathcal{H}(T) \approx 1.66 g_{*}^{1 / 2} T^{2} / M_{\mathrm{P}}$ is the Hubble rate during the radiation-dominated era, and $g_{*}$ is the number of relativistic degrees of freedom; the Planck mass is given by $M_{\mathrm{P}} \approx 1.2 \times$ $10^{19} \mathrm{GeV}$ [1]. In Eq. (A1), $\left\langle\sigma_{\text {wo }}\right\rangle$ is the thermally averaged washout cross section, $n_{\gamma}(T)$ is the photon number density, and $n_{i}(T)$ are the number densities for the initial-state particles relevant to the underlying process. A washout process is deemed ineffective if the condition

$$
\frac{\Gamma_{\text {wo }}(T)}{\mathcal{H}(T)}<1
$$

is satisfied.

To calculate $\left\langle\sigma_{\mathrm{wo}}\right\rangle$, we will follow the formalism of Ref. [51]. We have

$$
\left\langle\sigma_{\mathrm{wo}}\right\rangle=\frac{1}{\bar{n}_{1} \bar{n}_{2}} \int \frac{d^{3} p_{1} d^{3} p_{2}}{E_{1} E_{2}} f\left(E_{1}\right) f\left(E_{2}\right) w(s),
$$

where initial-state quantities are denoted by subscripts $\{1,2\}$, assuming $2 \rightarrow j$ processes; $j \geq 1$. In the above, $f\left(E_{1,2}\right)$ denote energy distributions of initial states, and $\bar{n} \equiv \int d^{3} p f(E)$. In the relativistic nondegenerate regime, relevant to our estimate here, we can assume a Boltzmann distribution for the initial states with $f(E)=e^{-E / T}$ to a good approximation [52]. This choice for $f(E)$ yields $\bar{n}=$ $8 \pi T^{3}$ in the massless limit, which is the case for our calculations below. The function $w(s)$, with $s=\left(p_{1}+p_{2}\right)^{2}$ being the center-of-mass energy squared, is defined by 


$$
w(s) \equiv \frac{1}{4} \int d \Phi \overline{|\mathcal{M}|^{2}}
$$

where the Lorentz-invariant phase space is given by

$$
d \Phi=(2 \pi)^{4} \delta^{(4)}\left(p_{1}+p_{2}-\sum_{j} p_{j}\right) \prod_{j} \frac{d^{3} p_{j}}{(2 \pi)^{3} 2 E_{j}}
$$

and $\overline{|\mathcal{M}|^{2}}$ denotes the squared amplitude for the underlying washout process, averaged and summed over the initialand final-state quantum numbers.

Since the function $w(s)$ is Lorentz invariant, we can calculate it in any convenient frame and recast the result as a function of $s$. Then, one may proceed to calculate $\left\langle\sigma_{\mathrm{wo}}\right\rangle$ in Eq. (A3) using

$$
s=M_{1}^{2}+M_{2}^{2}+2\left(E_{1} E_{2}-\left|\overrightarrow{p_{1}}\right|\left|\overrightarrow{p_{2}}\right| \cos \zeta\right),
$$

where $\zeta$ is the angle between initial momenta. For the washout processes relevant to our work, the initial states are massless: $M_{1}=M_{2}=0$.

In the model presented in this paper, we have assumed that quark couplings to heavy Higgs states $H_{a}$ are much larger than their couplings to leptons. Hence, we only consider $\Delta L$-erasing processes that involve at least one quark in the initial state. Also, we will not consider initial $\nu_{R}$ states, since they are not thermally populated in our scenario, in general. This is because the SM-like Higgs couplings to neutrinos $\sim 10^{-12}$, for the assumed Dirac neutrino masses, are too weak, and we are only interested in parameters for which washout through $H_{a}$ is ineffective.

With the above assumptions, the relevant $t$-channel processes we consider are $u L \rightarrow \nu_{R} Q, \quad \bar{Q} L \rightarrow \nu_{R} \bar{u}$, $Q L \rightarrow \nu_{R} d$, and $\bar{d} L \rightarrow \nu_{R} \bar{Q}$, where $(Q, L)$ denote (quark, lepton) doublets and $(u, d)$ denote up-type and down-type quark singlets, respectively. The $s$-channel processes are $\bar{d} Q \rightarrow \nu_{R} \bar{L}$ and $\bar{Q} u \rightarrow \nu_{R} \bar{L}$. As we have assumed nearly degenerate $H_{a}$ states, with the same couplings to various fermions, each of the preceding processes is summed over both contributions. In our calculation, we consider the case where only one generation of quarks and leptons-with Yukawa couplings $\lambda_{q}^{a}$ and $\lambda_{\nu}^{a}$, respectively-dominate the washout rate. For $T_{*}=100 \mathrm{GeV}$ and $m_{a}=10 \mathrm{TeV}$, we find $\lambda_{q}^{a} \lambda_{\nu}^{a} \lesssim 2.1 \times 10^{-4}$, as presented in Eq. (4).

\section{Asymmetry from modulus decay}

Let us assume a population of $\left\{H_{a}, H_{a}^{*}\right\}$ is nonthermally produced through the decays of a heavy modulus $\Phi$ of mass $m_{\Phi} ;\left\{H_{b}, H_{b}^{*}\right\}$ number density is taken to be negligible. The initial energy density of $H_{a}$ is given by $\rho_{a} \sim E_{a} n_{a}$, where $n_{a}$ is the $H_{a}$ number density and we have $E_{a} \sim m_{\Phi} / 2$. The decay of $H_{a}$ contributes to reheating of the Universe and $\rho_{a} \leq \rho_{R}$, with $\rho_{R}$ being the radiation energy density; $\rho_{R}=\left(\pi^{2} / 30\right) g_{*} T^{4}$, where $g_{*}$ is the number of relativistic degrees of freedom and $g_{*}=106.75$ in the $\mathrm{SM}$. We define the ratio

$$
r \equiv \frac{E_{a} n_{a}}{\rho_{R}}
$$

which satisfies $r \leq 1$. The $B-L$ abundance is then given by

$$
\frac{n_{B-L}}{s}=\frac{3 r T_{\mathrm{rh}} \varepsilon_{a}}{4 E_{a}},
$$

where $s=\left(2 \pi^{2} / 45\right) g_{*} T^{3}$ is the entropy density and the reheat temperature is $T_{\mathrm{rh}}$.

Since the new Higgs states are assumed to be heavy compared to $T_{\mathrm{rh}}$ (departure from equilibrium), the relevant degrees of freedom are those of the SM and, using the results of Ref. [53], one has

$$
\Delta B=\frac{28}{79} \Delta(B-L) .
$$

Given Eq. (A9), we then have

$$
\frac{n_{B}}{s}=\frac{21}{79}\left(\frac{r T_{\mathrm{rh}} \varepsilon_{a}}{E_{a}}\right)
$$

Using Eq. (1) and $T_{\mathrm{rh}} \sim 100 \mathrm{GeV}$, the above then yields Eq. (8).
[1] M. Tanabashi et al. (Particle Data Group), Phys. Rev. D 98, 030001 (2018).

[2] A. Sakharov, Sov. Phys. Usp. 34, 392 (1991).

[3] M. Fukugita and T. Yanagida, Phys. Lett. B 174, 45 (1986).

[4] M. Gell-Mann, P. Ramond, and R. Slansky, Conf. Proc. C 790927, 315 (1979), http://cds.cern.ch/record/133618.

[5] P. Minkowski, Phys. Lett. 67B, 421 (1977).
[6] R. N. Mohapatra and G. Senjanovic, Phys. Rev. Lett. 44, 912 (1980).

[7] P. Ramond, in International Symposium on Fundamentals of Quantum Theory and Quantum Field Theory (1979) [arXiv:hep-ph/9809459].

[8] H. Davoudiasl, I. M. Lewis, and M. Sullivan, Phys. Rev. D 101, 055010 (2020). 
[9] K. Dick, M. Lindner, M. Ratz, and D. Wright, Phys. Rev. Lett. 84, 4039 (2000).

[10] H. Murayama and A. Pierce, Phys. Rev. Lett. 89, 271601 (2002).

[11] H. Davoudiasl and I. Lewis, Phys. Rev. D 86, 015024 (2012).

[12] T. P. Cheng and M. Sher, Phys. Rev. D 35, 3484 (1987).

[13] Y. Grossman, Nucl. Phys. B426, 355 (1994).

[14] G. Cree and H. E. Logan, Phys. Rev. D 84, 055021 (2011).

[15] I. P. Ivanov, V. Keus, and E. Vdovin, J. Phys. A 45, 215201 (2012).

[16] V. Keus, S. F. King, and S. Moretti, J. High Energy Phys. 01 (2014) 052.

[17] M. P. Bento, H. E. Haber, J. C. Romão, and J. a. P. Silva, J. High Energy Phys. 11 (2017) 095.

[18] J. a. M. Alves, F. J. Botella, G. C. Branco, and M. Nebot, Eur. Phys. J. C 80, 710 (2020).

[19] H. E. Logan, S. Moretti, D. Rojas-Ciofalo, and M. Song, arXiv:2012.08846.

[20] W. Altmannshofer, J. Eby, S. Gori, M. Lotito, M. Martone, and D. Tuckler, Phys. Rev. D 94, 115032 (2016).

[21] D. Egana-Ugrinovic, S. Homiller, and P. Meade, Phys. Rev. Lett. 123, 031802 (2019).

[22] D. Egana-Ugrinovic, S. Homiller, and P. R. Meade, Phys. Rev. D 100, 115041 (2019).

[23] A. Pilaftsis, Phys. Rev. D 56, 5431 (1997).

[24] R. Gatto, G. Morchio, and F. Strocchi, Phys. Lett. 80B, 265 (1979).

[25] R. Gatto, G. Morchio, G. Sartori, and F. Strocchi, Nucl. Phys. B163, 221 (1980).

[26] G. Sartori, Phys. Lett. 82B, 255 (1979).

[27] A. Peñuelas and A. Pich, J. High Energy Phys. 12 (2017) 084.

[28] F. J. Botella, F. Cornet-Gomez, and M. Nebot, Phys. Rev. D 98, 035046 (2018).

[29] W. Rodejohann and U. Saldaña Salazar, J. High Energy Phys. 07 (2019) 036.

[30] A. Pilaftsis, Nucl. Phys. B504, 61 (1997).

[31] V. Andreev et al. (ACME Collaboration), Nature (London) 562, 355 (2018).

[32] A. Crivellin and L. Mercolli, Phys. Rev. D 84, 114005 (2011).

[33] B. Capdevila, A. Crivellin, S. Descotes-Genon, J. Matias, and J. Virto, J. High Energy Phys. 01 (2018) 093.
[34] M. Bona et al. (UTfit Collaboration), J. High Energy Phys. 03 (2008) 049.

[35] M. Bona (UTfit Collaboration), Proc. Sci., ICHEP2016 (2016) 149.

[36] R. Aaij et al. (LHCb Collaboration), Phys. Rev. Lett. 122, 231802 (2019).

[37] C. Lehner et al. (USQCD Collaboration), Eur. Phys. J. A 55, 195 (2019).

[38] J. Alwall, R. Frederix, S. Frixione, V. Hirschi, F. Maltoni, O. Mattelaer, H. S. Shao, T. Stelzer, P. Torrielli, and M. Zaro, J. High Energy Phys. 07 (2014) 079.

[39] N. D. Christensen and C. Duhr, Comput. Phys. Commun. 180, 1614 (2009).

[40] A. Alloul, N. D. Christensen, C. Degrande, C. Duhr, and B. Fuks, Comput. Phys. Commun. 185, 2250 (2014).

[41] G. Aad et al. (ATLAS Collaboration), J. High Energy Phys. 03 (2020) 145.

[42] A. M. Sirunyan et al. (CMS Collaboration), J. High Energy Phys. 05 (2020) 033.

[43] T. Golling et al., CERN Yellow Rep. 441 (2017).

[44] M. E. Peskin and T. Takeuchi, Phys. Rev. Lett. 65, 964 (1990).

[45] M.E. Peskin and T. Takeuchi, Phys. Rev. D 46, 381 (1992).

[46] R. Barbieri, L. J. Hall, and V. S. Rychkov, Phys. Rev. D 74, 015007 (2006).

[47] H. E. Haber and D. O'Neil, Phys. Rev. D 83, 055017 (2011).

[48] A. Ahriche, G. Faisel, S.-Y. Ho, S. Nasri, and J. Tandean, Phys. Rev. D 92, 035020 (2015).

[49] A. Abada et al. (FCC Collaboration), Eur. Phys. J. Special Topics 228, 755 (2019).

[50] Y. Cui, L. Randall, and B. Shuve, J. High Energy Phys. 04 (2012) 075.

[51] M. Srednicki, R. Watkins, and K. A. Olive, Nucl. Phys. B310, 693 (1988).

[52] E. W. Kolb and M. S. Turner, The Early Universe, Vol. 69 (Frontiers in Physics, 1990), ISBN 978-0-201-62674-2, https:/ui.adsabs.harvard.edu/abs/1990eaun.book.....K/ abstract.

[53] J. A. Harvey and M. S. Turner, Phys. Rev. D 42, 3344 (1990). 